\title{
AN INTERESTING CASE OF VALVULAR HEART DISEASE: A CASE REPORT
}

\author{
Anusuya Meganathan ${ }^{1}$, Saranya Devi Kanagaraj² \\ ${ }^{1}$ Associate Professor, Department of General Medicine, Chengalpattu Medical College and Hospital. \\ 2Junior Resident, Department of General Medicine, Chengalpattu Medical College and Hospital.
}

\begin{abstract}
A 24-year-old male presented with symptoms of acute onset altered sensorium and seizures. He was diagnosed with hypertensive encephalopathy and retinopathy. He was a candidate of double valve replacement surgery, which he underwent 3 years back. Further workup with renal artery Doppler revealed unilateral renal artery stenosis with extensive collateral circulation. Patient underwent a complete CT aortogram, which revealed large vessel vasculitis. We report this case as it is a rare initial presentation of Takayasu arteritis in a male to involve double valves of the heart.
\end{abstract}

\section{KEYWORDS}

Takayasu Arteritis, Valvular Heart Disease, Renovascular Hypertensive with Double Valve Replacement, Congestive Cardiac Failure and Takayasu Arteritis, Regurgitant Heart Lesion in Takayasu Arteritis.

HOW TO CITE THIS ARTICLE: Meganathan A, Kanagaraj SD. An interesting case of valvular heart disease: a case report. J. Evolution Med. Dent. Sci. 2016;5(46):2938-2940, DOI: 10.14260/jemds/2016/683

\section{INTRODUCTION}

A 24 year male patient presented with history of progressive headache for a week, which culminated in altered behaviour and 2 episodes of seizures for which the patient was managed symptomatically. The seizures were GTCS in type with frothing from mouth and tongue bites during the episode. There was no history of fever/rash, trauma, vomiting and loose stools. There was no history of chest pain, palpitations or shortness of breath - PND or orthopnoea. No history of bleeding diathesis or bladder and bowel incontinence.

His past history showed that he was a candidate of double valve replacement done in May 2010. Patient had history of progressive dyspnoea for two long years from 2008, when he was evaluated for his complaints in 2010 and found to have severe mitral and aortic regurgitation with pulmonary hypertension. He presented with congestive heart failure in a private hospital. He was treated with double valve replacement of the mitral and aortic apparatus with $27 \mathrm{~mm}$ and $19 \mathrm{~mm}$-FK Chitra valves. Post-operative period was uneventful. Patient was started on oral anticoagulants and anti-failure drugs. Discharged with advice of regular follow-up of PT/INR. Patient's last INR done 3 years back was 1.25. He was on T. Acitrom $2 \mathrm{mg}$.

Patient presented one year later with history of claudication pain of both lower limbs to ER of the same hospital. He was advised arterial Doppler of both his lower limbs, as both his lower limb dorsalis pedis pulses were weak. Patient did not take the advice and continued the drugs, one year later following which he presented with seizures and altered behaviour at our hospital.

He was not a known hypertensive or diabetic, no history of tuberculosis in the past. History of wheeze since childhood, for which the patient was put on salbutamol nebulization and bronchodilators.

Financial or Other, Competing Interest: None.

Submission 23-03-2016, Peer Review 18-05-2016,

Acceptance 24-05-2016, Published 09-06-2016.

Corresponding Author:

Dr. Anusuya Meganathan,

\#351, Konnur High Road,

Ayanavaram,

Chennai-600023.

E-mail: anusuyarubi@hotmail.com

DOI: $10.14260 /$ jemds/2016/683
He is the second child born out of a non-consanguineous marriage - pre-natal and post-natal history uneventful, studied up to 10th standard, works in a grocery shop. No history of similar complaints in other family members. $\mathrm{He}$ is unmarried, non-alcoholic, non-smoker with no history of substance abuse. He is a non-vegetarian with no history of sleep disturbances with normal bladder and bowel habits.

On examination patient was irritable, well-built, wellnourished with no pallor or icterus, no dyspnoea or tachypnoea with no pedal oedema, clubbing, cyanosis or generalised lymphadenopathy. No markers of Tuberculosis, Rheumatic fever or HIV. His pulse rate was $96 / \mathrm{min}$ with diminished right radial pulse and both femorals, popliteals, dorsalis pedis and posterior tibial artery. His initial blood pressure recordings were $210 / 160$ in the left upper limb, $180 / 160$ in the right upper limb and both lower limbs.

On auscultation, the metallic click of prosthetic heart valves was heard. The lungs were clear. There was no organomegaly or vascular bruit/hum on palpation and auscultation of abdomen respectively. There was no focal neurological deficit except for the irritability. His funduscopic examination showed a grade 2 hypertensive retinopathy.

Basic investigations were done. His total count was 14,800 with ESR of 4 and Haemoglobin of 14.7. His blood sugar was normal with creatinine of $1.7 \mathrm{mg} \%$ and urea of $36 \mathrm{mg} \%$. CPK elevated, normalized after adequate hydration and his Creatinine level was $0.9 \mathrm{mg} \%$. His liver function tests were normal. ICTC and VDRL were negative. Mantoux was negative. CXR was normal showing the prosthetic valves. His lipid profile was within normalcy.

PT/INR done 1.25. T. Acitrom was increased to $3 \mathrm{mg}$ with heparin bridging. His initial CT Brain was normal.

Cardiac evaluation was done that showed normally functioning cardiac prosthesis in situ without evidence of clot of thrombi with concentric LVH.

CRP levels were elevated and ASO titre was within normal limits. ANA, RA factor and ANCA were negative.

MRI was taken after confirming the valve compatibilitysuggested diffuse TI and T2 hyperintensities with diffuse attenuation of left internal carotid artery caliber and hypoplastic left transverse sinus. 
Carotid Doppler - diffusely attenuated caliber of left internal carotid artery at the level of bifurcation with reduced velocity.

Renal artery Doppler done - ostial stenosis of left renal artery and was suggested renal angiogram.

Doppler of both lower limbs revealed bilateral iliac artery stenosis. Aortogram done - occlusion of cervical segment of left internal carotid artery just beyond the origin with faint reformation of supraglenoid segment of internal carotid artery with narrowing of infrarenal aorta $4 \mathrm{~cm}$ below the origin of renal arteries with reformation of bilateral external iliac artery by collaterals. Renal veins single on both sides with retroperitoneal varices and normal CT urogram.

A diagnosis of Takayasu arteritis was made. Patient started on T. Methotrexate and oral steroids with folic acid supplementation. Renal artery stenting advised by vascular surgeon.

This case is presented for involvement of double valves of heart with regurgitant lesions, which is rare in a young male with Takayasu arteritis.

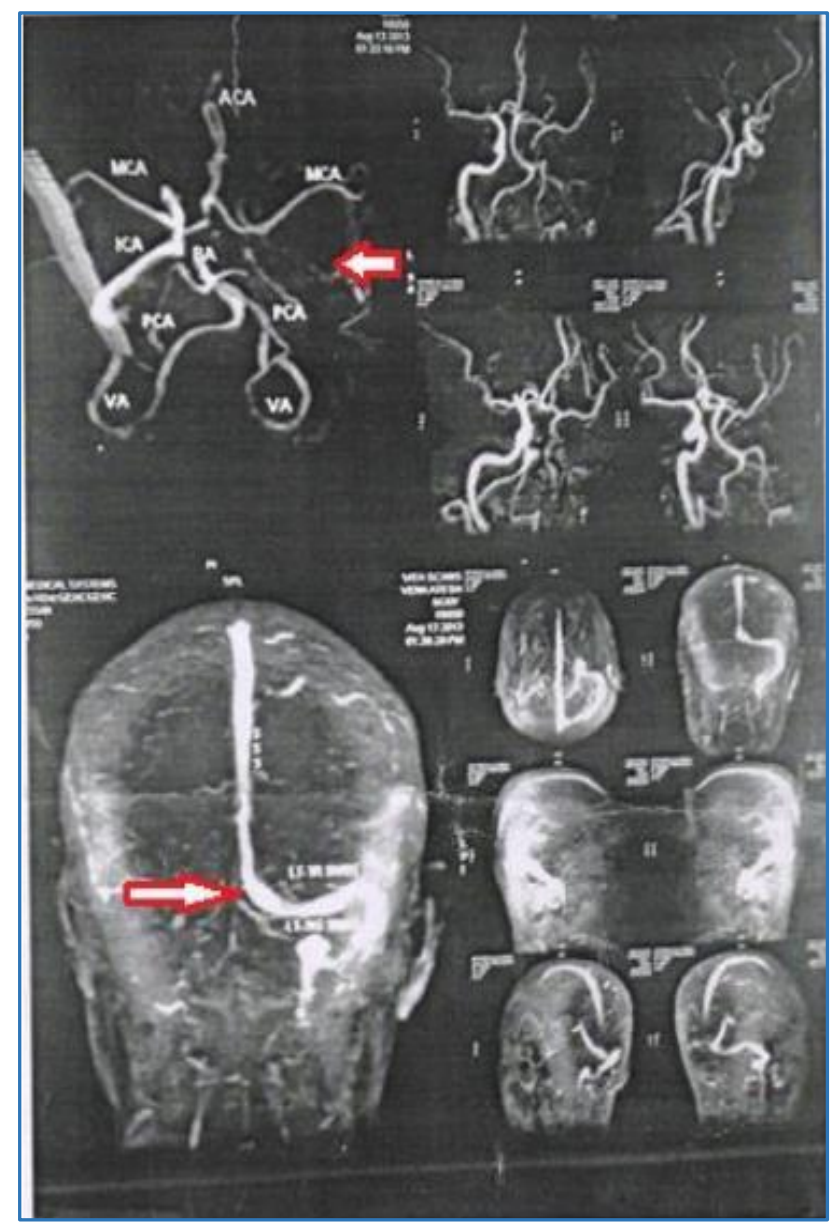

Fig. 1: MR Angiogram showing Complete Occlusion of Left Internal Carotid Artery

\section{REVIEW OF LITERATURE}

Takayasu arteritis is an inflammatory and stenotic disease of medium and large sized arteries characterised by a strong predilection for the Aorta and its branches.[1] Also known as aortic arch syndrome, pulseless disease, reverse coarctation of aorta, Martorell disease. Dr. Mikito Takayasu described the retinal changes of the disease in 1908 at the Annual Ophthalmology Society meeting in Japan and his abstract was

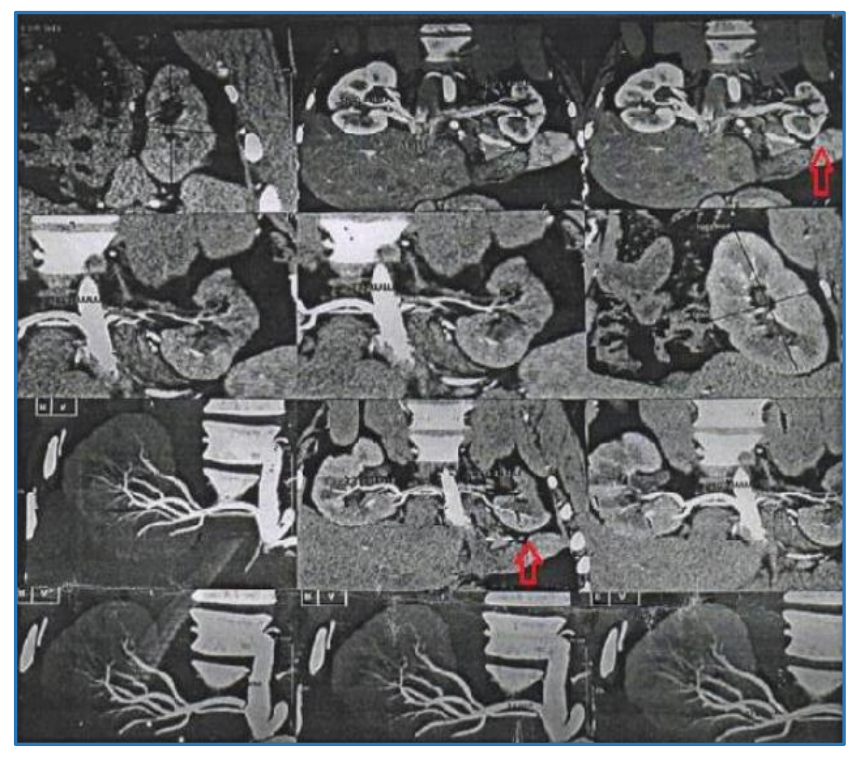

Fig. 2: CT Angiogram showing Left Contracted Kidney with Thinned Out Left Renal Artery

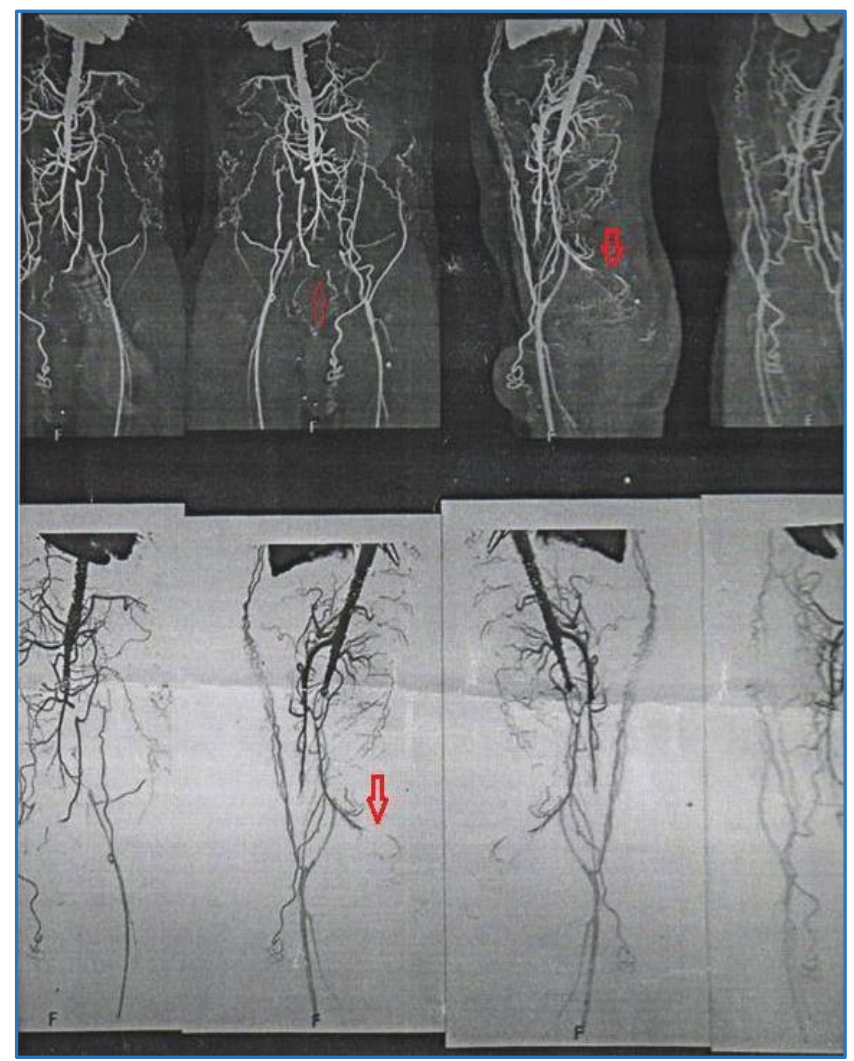

Fig. 3: CT Angiogram showing Proximal Left Common Iliac Artery Occlusion

subsequently published in 1908 . The condition is now called Takayasu arteritis in his honour.

Incidence and prevalence - Uncommon disease. Annual incidence rate 1.2-2.6 cases/million.[2] Prevalent in adolescent and young women in Japan and India. Takayasu arteritis is observed more frequently in patients of Asian or Indian descent. Japanese have a higher incidence of aortic arch involvement. India report higher incidences of abdominal 
involvement. Takayasu arteritis is the most common cause of reno-vascular hypertension in India, $80 \%$ of patients-women; however, the high female-to-male ratio seems to decrease in west of Japan. In India, the female-to-male ratio is as low as 1.6:1.

Pathology is pan inflammatory arteritis with fibrosis and scarring producing intimal thickening.[3]

Symptoms can be Headache (50\%-70\%), Malaise (35\%65\%), Arthralgias (28\%-75\%), Fever (9\%-35\%), Weight loss (10\%-18\%).

Cardiac and vascular manifestations can be in the form of bruit with the most common location being the carotid artery $(80 \%)$. Blood pressure difference of extremities is $(45 \%$ $69 \%)$. Claudication (38\%-81\%), Carotidynia or vessel tenderness (13\%-32\%), Hypertension (28\%-53\%) (58\% with renal artery stenosis in one series), Aortic regurgitation (20\%$24 \%)$, Raynaud's syndrome $(15 \%)$. Pericarditis $(<8 \%)$, Congestive heart failure $(<7 \%)$, Myocardial infarction $(<3 \%)$.

Neurological symptoms like Headache (50\%-70\%), Visual disturbance (16\%-35\%) can occur. Strong association with common carotid and vertebral artery disease, Stroke $(5 \%$ 9\%), Transient ischaemic attacks (3\%-7\%), Seizures $(0 \%-$ 20\%).

Particular attention to peripheral pulses, blood pressure in all 4 extremities and an ophthalmologic examination. The most discriminatory finding is a systolic blood pressure difference (>10 mmHg) between the arms. Hypertension due to renal artery involvement is found in approximately $50 \%$ of patients. Absent or diminished pulses are the clinical hallmark of Takayasu arteritis, but pulses are normal in many patients and upper limbs are affected more often than lower limbs. Carotidynia may be present. Bruits are often noted. Aortic regurgitation is a common finding.

Ophthalmologic examination may show retinal haemorrhages, cotton-wool exudates, venous dilatation and beading, micro-aneurysms of peripheral retina, optic atrophy, vitreous haemorrhage and classic wreath-like peri-papillary arteriovenous anastomoses (Extremely rare).

Skin changes resembling erythema nodosum or ulcerating nodular lesions may be seen.

\section{ACR Criteria.[4]}

\section{3 of 6 Necessary}

1. Age $<40$ years of onset.

2. Claudication of extremities.

3. Decreased pulsation in one/both brachial arteries.

4. Difference of at least $10 \mathrm{mmHg}$ SBP between the arms.

5. Bruit over one/both subclavian artery/abdominal aorta.

6. Arteriographic narrowing/occlusion of entire abdominal aorta, its primary branches or large arteries of both UL and LL.

\section{Tests}

Arteriogram, Angiogram, Complete Blood Count (CBC), CReactive Protein (CRP), Electrocardiogram (ECG), Erythrocyte Sedimentation Rate (ESR), Magnetic Resonance Angiography (MRA), Magnetic Resonance Imaging (MRI), Ultrasound, Xray of the chest.

\section{Classification}

Takayasu Arteritis can be Divided into 6 Types Based on Angiographic Involvement as follows:

Type I: Branches of the aortic arch.

Type IIa: Ascending aorta, aortic arch and its branches.
Type IIb: Ila region plus thoracic descending aorta.

Type III: Thoracic descending aorta, abdominal aorta, renal arteries or a combination.

Type IV: Abdominal aorta, renal arteries or both.

Type V: Entire aorta and its branches.

\section{What is Active Disease?}

New onset or worsening of two or more of the following features indicates active disease:

1. Systemic features, such as fever and arthralgias (No identified cause), erythrocyte sedimentation rate.

2. Features of vascular ischemia or inflammation such as claudication, diminished or absent pulse, bruit, carotidynia or asymmetric blood pressure in either upper or lower limbs (Or both) and typical angiographic features.

\section{Treatment}

Takayasu arteritis is difficult, but patients who do have the right treatment can see positive results. Early detection is important. Most patients are treated with steroids and immunosuppressive drugs. Surgery is reserved for complications caused by narrowed arteries. Surgery to bypass narrowed arteries - angioplasty or stent placement - may be needed to supply blood or open up the constriction.[5]

\section{Morbidity and Mortality}

Takayasu arteritis is a chronic relapsing and remitting disorder; 10-year survival rate is approximately 90\%; however, this rate is reduced in the presence of major complications.

Complications of Takayasu arteritis include valvular heart disease, stroke, heart failure, retinopathy and renovascular hypertension. The 5- and 10-year survival rates are approximately $69 \%$ and $36 \%$ respectively, in patients with two or more complications. The 5- and 10-year survival rates associated with one or fewer complications are $100 \%$ and $96 \%$, respectively.

A 2008 study assessing quality of life with Takayasu arteritis shows worse scores for physical and mental health compared with many other chronic diseases associated with peripheral vascular disease. Disease remission is the only factor that positively influences both physical and mental quality of life.

\section{REFERENCES}

1. Gary S Firestein, Kelley's textbook of rheumatology ninth edition elesvier saunders, United states, chapter no 88, ISBN 978-1-4377-1738-9, 2013;2:1461-81.

2. Dennis L Kasper, Harrison's principles of internal medicine $19^{\text {th }}$ ed. McGraw Hill education, United states, chapter no 385, ISBN 978-0-07-180216-1, 2015;2:217980.

3. Kinare SG, Gandhi MS, Deshpande J. Non-specific aortoarteritis Takayasu's disease. Pathology and Radiology. Mumbai: Quest publications 1988.

4. Arend WP, Michel BA, Bloch DA, et al. The American college of rheumatology 1990 criteria for the classification of Takayasu arteritis. Arthritis rheum 1990;33(8):1129-34.

5. Pokrovskii AV. Nonspecific aorto-arteritis (classification and surgical treatment). Kardiologiia 1986;26(3):5-12. 